ISSN 0103-9954

\title{
EFEITOS DE DIFERENTES TIPOS DE CONTROLE DE PLANTAS INFESTANTES SOBRE A MIRMECOFAUNA EM Eucalyptus grandis
}

\author{
EFFECTS OF DIFFERENT TYPES OF WEED CONTROL ON THE ANT FAUNA IN \\ Eucalyptus grandis
}

\author{
Jardel Boscardin ${ }^{1}$ Ervandil Corrêa Costa ${ }^{2}$ Juliana Garlet ${ }^{3}$ Leonardo Mortari Machado ${ }^{4}$ \\ Dayanna do Nascimento Machado ${ }^{5}$ Leandra Pedron ${ }^{5}$ Lisandro Cunha Bolzan ${ }^{5}$
}

\begin{abstract}
RESUMO
Este estudo teve como objetivo avaliar os efeitos de diferentes tipos de controle de plantas infestantes sobre a mirmecofauna em um plantio inicial de Eucalyptus grandis. Para tanto, em março de 2011 foi realizado o início da implantação da cultura, em uma área localizada no município de Santa Maria - RS. Os seis tratamentos constituíram-se em controle químico total de plantas infestantes, na linha e na entrelinha de plantio, com glifosato (T1); controle químico total de plantas infestantes, na linha de plantio, com glifosato (T2); controle químico de monocotiledôneas na linha e entrelinha de plantio, com setoxidim (T3); controle químico de dicotiledôneas na linha e entrelinha de plantio, com bentazona (T4); controle químico total de plantas infestantes em faixa de um metro paralela à linha de plantio, com glifosato, e de um metro na parte central da entrelinha, sem controle (T5); e testemunha, sem controle de plantas infestantes (T6). O levantamento da mirmecofauna foi realizado no período de um ano utilizando-se três métodos de coleta: isca atrativa, armadilha de solo e funil de Berlese, com seis repetições por tratamento, em cada data de coleta. Nesse período foram coletadas 46.675 formigas, distribuídas em 37 espécies, não sendo verificada diferença significativa entre o total de espécimens coletados. $\mathrm{Na}$ área do tratamento constituído pelo controle de plantas infestantes somente na linha de plantio verificou-se eficiência amostral de $99,0 \%$ e $S_{\mathrm{obs}}=35$. Entre os índices de Diversidade de Shannon $\left(H^{\prime}\right)$, destacou-se o valor encontrado para a área do tratamento $\mathrm{T} 2\left(H^{\prime}=1,34\right)$ em detrimento dos valores das áreas nas quais foram instalados os tratamentos T1 $\left(H^{\prime}=1,25\right)$ e T5 $\left(H^{\prime}=1,23\right)$. Havendo assim, uma maior coexistência de espécies de formigas entre as áreas com estrutura florística menos alterada e entre as áreas mais simplificadas. Não tendo sido verificada correlação significativa $(r=0,0463)$ entre a riqueza de espécies de formigas coletadas e o número de plantas infestantes encontradas ao final do experimento. Assim, conclui-se que os efeitos indiretos da ação dos herbicidas afetam mais a composição local de espécies de formigas do que sua riqueza.
\end{abstract}

Palavras-chave: entomologia florestal; Formicidae; matocompetição; plantio inicial.

\section{ABSTRACT}

The aim of this study was to evaluated the effects of different types of weed control on the ant fauna in an

1 Engenheiro Florestal, Doutorando do Programa de Pós-Graduação em Engenharia Florestal, Centro de Ciências Rurais, Universidade Federal de Santa Maria, Av. Roraima, 1000, CEP 97105-900, Santa Maria (RS), Brasil. boscardinj@gmail.com

2 Engenheiro Agrônomo, Dr., Professor do Programa de Pós-Graduação em Engenharia Florestal, Centro de Ciências Rurais, Universidade Federal de Santa Maria, Av. Roraima, 1000, CEP 97105-900, Santa Maria (RS), Brasil.ervandilc@gmail.com

3 Engenheira Florestal, Dra., Professora Adjunta da Faculdade de Ciências Biológicas e Agrárias, Universidade do Estado do Mato Grosso, Av. Perimetral Rogério Silva, s/n, CEP 78580-000, Alta Floresta (MT), Brasil. julianagarlet@yahoo.com.br

4 Engenheiro Florestal, Doutorando do Programa de Pós-Graduação em Engenharia Florestal, Centro de Ciências Rurais, Universidade Federal de Santa Maria, Av. Roraima, 1000, CEP 97105-900, Santa Maria (RS), Brasil. leonardomortarimachado@yahoo.com.br

5 Engenheira (o) Florestal, Mestranda do Programa de Pós-Graduação em Engenharia Florestal, Centro de Ciências Rurais, Universidade Federal de Santa Maria, Av. Roraima, 1000, CEP 97105-900, Santa Maria (RS), Brasil. dayanasmac@gmail.com/leandra_pedron@hotmail.com/lisandro.bolzan@hotmail.com 
initial planting of Eucalyptus grandis. Therefore, in march of 2011 was performed the implantation of culture, in an area located in the city of Santa Maria - RS. The six treatments were constituted by the total chemical control of weeds in row and interrow planting, with glyphosate (T1); total chemical control of weeds in the crop row, with glyphosate (T2); chemical control of monocotyledonous in row and interrow planting, with sethoxydim (T3); chemical control of dicotyledonous line interrow and planting, with bentazone (T4); total chemical control of weeds in a yard track parallel to the row, with glyphosate, and a meter in the central part of interrow without control (T5); and without control weeds (T6). The survey of the ant fauna was conducted in one year using three collection methods: attractive bait, trap soil and Berlese funnel, with six replicates per treatment in each collection date. In this period were collected 46,675 ants, belonging to 37 species, there being no significant difference between the total specimens collected. In the area of treatment consists of the control of weeds in the crop row only verified efficiency of $99.0 \%$ and sample $S_{\text {obs }}=35$. Among the indices of Shannon Diversity $\left(H^{\prime}\right)$, highlighted the value found for the area of treatment T2 $\left(H^{\prime}=1.34\right)$ at the expense of the values of the areas where they were installed the T1 $\left(H^{\prime}=1,25\right)$ and T5 $\left(H^{\prime}=1,23\right)$. Having thus a higher co-existence of ant species between the areas with changed structure less flora, and between the areas most simplified. There were significant correlation $(r=0.0463)$ between the species richness of ants collected and the number of weeds found at the end of the experiment. Thus, we conclude that the indirect effects of the herbicides affect more the local composition ant species than his richness.

Keywords: forest entomology; Formicidae; weed competition; initial planting.

\section{INTRODUÇÃO}

Plantios com espécies florestais possuem importância econômica e socioambiental para o Brasil, contribuindo para a diminuição da supressão das matas nativas. Segundo dados da Associação Brasileira de Produtores de Florestas Plantadas (ABRAF, 2012), os plantios comerciais com espécies do gênero Eucalyptus representam a maior parcela de espécies florestais plantadas no país.

A implantação constitui-se em uma etapa decisiva para o sucesso de um empreendimento florestal, entre os cuidados a serem tomados nessa fase, destaca-se o controle de plantas infestantes ou daninhas.

De acordo com Perrando (2008), entre os herbicidas utilizados no controle de plantas infestantes, o glifosato é utilizado em larga escala por tratar-se de um herbicida pós-emergente que facilita o manejo de plantas infestantes em áreas de cultivo mínimo, assegurando o potencial produtivo da cultura florestal, minimizando o custo de produção e os impactos ao ambiente. O controle de plantas infestantes com glifosato, em aplicações dirigidas, é intenso nos dois primeiros anos de implantação da cultura do eucalipto, podendo, em caso de reinfestação, estender-se para quase todo o ciclo da cultura (TUFFI SANTOS, 2006).

Segundo Ferreira et al. (2010), existem vários questionamentos acerca dos efeitos negativos de herbicidas, como o glifosato, no crescimento e desenvolvimento do eucalipto, no manejo de rebrotas e de espécies de difícil controle, bem como na suscetibilidade da cultura a doenças e ataques de pragas.

Em plantios homogêneos, como do eucalipto, os insetos herbívoros exibem taxas de colonização mais elevadas, maior potencial reprodutivo, maior tempo de permanência, menos barreiras ao encontro do hospedeiro e menores taxas de mortalidade por predadores naturais.mais baixas. Porém, deve-se adotar uma postura cautelosa e flexível, sem generalizações, pois as respostas dos herbívoros à diversidade de vegetação não são uniformes e nem sempre podem ser explicadas através da diversidade por si só (ALTIERI; SILVA; NICHOLLS, 2003).

Para Wardle et al. (2001), os efeitos deletérios na densidade da fauna edáfica são incertos e, parecem estar mais ligados à simplificação do habitat, através da retirada da cobertura vegetal viva proporcionada pelas plantas infestantes, do que à intoxicação direta da fauna pelo herbicida.

Dentre os insetos que forrageiam sobre o solo estão as formigas, que se constituem como um dos táxons mais importantes em termos de biomassa ou abundância relativa local (HÖLLDOBLER; WILSON, 1990). Segundo Alonso (2000), os padrões de riqueza e diversidade de espécies de formigas podem ser correlacionados com táxons, 
que possuam exigências similares quanto a nidificação e a alimentação, que são afetados por fatores semelhantes, ou com os quais as formigas estabeleçam interações.

Além disso, formigas são tidas como potenciais indicadores da qualidade ambiental de um ecossistema, por apresentarem muitos dos fatores exigidos aos bioindicadores. Conforme descrito por Majer (1983), tais fatores podem ser: abundância local alta, riqueza de espécies local e global alta, muitos táxons especializados, facilidade de serem amostradas, facilidade de serem separadas em morfoespécies e sensibilidade às mudanças nas condições do ambiente.

Assim, a partir do levantamento e análise da diversidade local de formigas, torna-se possível avaliar os efeitos ocasionados por tratos silviculturais aplicados na implantação e condução de um plantio florestal. Neste sentido, este trabalho objetivou avaliar a fauna de formigas em um plantio inicial de Eucalyptus grandis Hill ex Maiden, submetido a diferentes tipos de controle de plantas infestantes, no município de Santa Maria, Rio Grande do Sul.

\section{MATERIAIS E MÉTODOS}

\section{Localização e caracterização da área experimental}

O estudo foi conduzido na Unidade de Pesquisa Florestal pertencente à Fundação Estadual de Pesquisa Agropecuária (FEPAGRO) (2940’31'S' 5354'45’W) no município de Santa Maria - RS, localizado na região fisiográfica da Depressão Central do estado do Rio Grande do Sul, com altitude média de 130 metros.

O clima da região, segundo Köppen, é do tipo Cfa, com temperatura média do mês mais frio compreendida entre $-3^{\circ} \mathrm{C}$ e $18^{\circ} \mathrm{C}$, e temperatura média do mês mais quente superior a $22^{\circ} \mathrm{C}$. A precipitação média anual é de $1.770 \mathrm{~mm}$, sem estiagens (MORENO, 1961).

$\mathrm{O}$ solo da região pertence à Unidade de Mapeamento São Pedro e é classificado como Argissolo Vermelho distrófico arênico (STRECK et al., 2008). A área apresentava, inicialmente, cobertura vegetal composta, predominantemente, por espécies de gramíneas de baixo porte, e estava sendo utilizada para pecuária nos últimos 20 anos.

\section{Delineamento experimental}

O Delineamento experimental escolhido foi o Inteiramente Casualizado (DIC), sem restrições, com distribuição aleatória dos seis tratamentos, através de sorteio. A área de cada tratamento era de meio hectare.

Os tratamentos analisados foram constituídos por controle químico total de plantas infestantes, na linha e na entrelinha do plantio, com ingrediente ativo glifosato, na dosagem de 3 litros por hectare $\left(\mathrm{L} \mathrm{ha}^{-1}\right)$ do produto comercial, e volume de calda de $200 \mathrm{~L}^{-1}$ (T1); controle químico total de plantas infestantes, na linha de plantio, com ingrediente ativo glifosato, na dosagem de $3 \mathrm{~L} \mathrm{ha}^{-1}$, e volume de calda de $200 \mathrm{~L} \mathrm{ha}^{-1}$ (T2); controle químico de monocotiledôneas na linha e entrelinha de plantio, com ingrediente ativo setoxidim, na dosagem de $1,25 \mathrm{~L} \mathrm{ha}^{-1}$, e volume de calda de $200 \mathrm{~L} \mathrm{ha}^{-1}$ (T3); controle químico de dicotiledôneas na linha e entrelinha do plantio, com ingrediente ativo bentazona na dosagem de 1,2 $\mathrm{L} \mathrm{ha}^{-1}$, com adição de $1 \mathrm{~L} \mathrm{ha}^{-1}$ de adjuvante, e volume de calda de $150 \mathrm{~L} \mathrm{ha}^{-1}$ (T4); controle químico total de plantas infestantes em faixa de um metro paralelo à linha de plantio, e um metro na parte central da entrelinha, sem controle, com ingrediente ativo glifosato na dosagem de $3 \mathrm{~L} \mathrm{ha}^{-1}$, e volume de calda de $200 \mathrm{~L} \mathrm{ha}^{-1}$ (T5); e testemunha, sem controle de plantas infestantes e nenhuma forma de limpeza na área (T6).

\section{Instalação do experimento}

Para o plantio, foi adotado cultivo mínimo, com revolvimento do solo sob sistema mecanizado com grade rotativa (profundidade média de $30 \mathrm{~cm}$ ).

As mudas de Eucalyptus grandis, com altura média de 30 centímetros $(\mathrm{cm})$, provenientes da produção da própria FEPAGRO, foram transplantadas em 11 de agosto de 2011, em espaçamento $3 \times 2$ (três metros na entrelinha e dois metros na linha), nos três hectares de área (densidade de 1.667 mudas ha ${ }^{-1}$ ), totalizando aproximadamente 5.000 mudas. O replantio, na proporção de 26,9 por cento (\%), aproximadamente 1.500 mudas, foi realizado em setembro do mesmo ano.

A aplicação dos herbicidas foi realizada utilizando-se pulverizador costal manual de pressão constante, com barras munidas de dois ou quatro bicos do tipo leque. Após o transplante das mudas, na linha de plantio, os herbicidas foram aplicados 
com chapéu de Napoleão sobre o bico. A aplicação foi sequencial: primeiramente em área total, antes do plantio, respectivamente, a primeira $(01 / 04 / 2011)$ e a segunda (30/06/2011) aplicações, e após o transplante, a terceira aplicação (21/10/2011), na linha de plantio.

\section{Avaliação da mirmecofauna}

As coletas da mirmecofauna foram realizadas antes e depois da aplicação dos herbicidas nas áreas, que ocorreram no período de março de 2011 a fevereiro de 2012. De acordo com Lopes e Vasconcelos (2008), quando se pretende realizar um inventário completo da fauna de formigas, deve-se utilizar mais de um método de coleta. Diante do exposto, buscou-se amostrar de forma ampla a diversidade de formigas encontradas no local, e para isso utilizaram-se três métodos de coletas, descritos na sequência.

O método de coleta utilizando isca atrativa (IA) à base de proteína animal foi realizado quinzenalmente totalizando 23 amostragens. As armadilhas constituíram-se de um papel-filtro com 36 centímetros quadrados $\left(\mathrm{cm}^{2}\right)$, no qual foi depositada uma porção de aproximadamente um centímetro cúbico $\left(\mathrm{cm}^{3}\right)$ de isca à base de patê de fígado de frango, segundo Boscardin et al. (2011).

As iscas atrativas foram dispostas a 10 metros (m) de distância entre si, conforme sugere Sarmiento-M (2003), no interior da parcela de cada tratamento, em dois transectos, sendo um transecto na linha de plantio e outro na entrelinha, distantes $10 \mathrm{~m}$ um do outro. Totalizando, assim, seis armadilhas distribuídas por tratamento, totalizando 36, em cada data de coleta. Após 60 minutos de exposição, as armadilhas foram recolhidas e depositadas em recipientes plásticos, de 80 mililitros $(\mathrm{mL})$, contendo álcool $70^{\circ} \mathrm{GL}$, etiquetados com identificação, e transportados até o Laboratório de Entomologia Florestal do Departamento de Defesa Fitossanitária (UFSM).

A partir da utilização do método com armadilhas de solo (AS), modelo pitfall, foram realizadas coletas a cada 20 dias, totalizando 20 amostragens. As armadilhas constituíram-se de um recipiente cilíndrico de $10 \mathrm{~cm}$ de altura, enterrado no solo até a borda, sendo a área de captura de $176,7 \mathrm{~cm}^{2}$.

As armadilhas de solo continham como líquido conservante, uma solução contendo 200 $\mathrm{mL}$ de água, 15 gramas (g) de cloreto de sódio e
$2 \mathrm{~mL}$ de detergente. Utilizaram-se seis armadilhas de solo, por tratamento, totalizando 36 , em cada data de coleta, distribuídas da mesma forma que o método de coleta com isca atrativa à base de proteína animal. Após 48 horas de exposição, todo o material coletado nas armadilhas foi recolhido em recipientes plásticos $(80 \mathrm{~mL})$, etiquetados e transportados até o Laboratório de Entomologia Florestal (UFSM).

Já a extração das formigas com funil de Berlese (FB) foi realizada mensalmente, totalizando 11 amostragens. Com o auxílio de uma sonda circular, de capacidade volumétrica de $785 \mathrm{~cm}^{3}$, retiraram-se, aleatoriamente, seis amostras de solo, por tratamento, em cada data de coleta. Em seguida as amostras embaladas, identificadas foram transportadas até o Laboratório de Entomologia Florestal (UFSM).

Em laboratório, as amostras foram depositadas nos funis extratores de Berlese, permanecendo por um período de quatro dias. Sob cada funil, foi colocado um recipiente de plástico com capacidade de $100 \mathrm{~mL}$, contendo álcool a $70^{\circ} \mathrm{GL}$. O processo de extração das formigas ocorreu pela incidência de calor propiciado pelas lâmpadas incandescentes de 60 watts dispostas sobre a abertura superior dos funis de Berlese, que fez com que as formigas migrassem em direção do recipiente plástico, sendo então capturadas (BESTELMEYER et al., 2000). A temperatura do solo no funil permaneceu em torno de 30 graus.

As amostras coletadas a campo, com iscas e armadilhas de solo e funil de Berlese, foram submetidas a triagem no laboratório, com o auxílio de um estereoscópio. Em seguida, as formigas encontradas foram identificadas em nível de gênero e separadas em morfoespécies, segundo a chave de identificação de Bolton (1994).

As morfoespécies receberam um código de identificação e foram depositadas em microtubos do tipo eppendorf. Estes, foram enviados ao Laboratório de Mirmecologia do Centro de Pesquisas do Cacau, Ilhéus, Bahia, para identificação, cuja série ficou tombada na coleção referência da mesma instituição, registrada sob o número \#5683.

\section{Análise dos parâmetros ecológicos}

A abundância total de formigas compreendeu o número de formigas e/ou espécimens coletados nos três métodos de amostragem durante todo o período do estudo.

A riqueza observada de espécies $\left(S_{\text {obs }}\right)$ de 
formigas foi obtida pelo somatório do número de espécies coletadas em cada amostra, no período compreendido de março de 2011 a fevereiro de 2012. Para calcular a riqueza estimada $\left(S_{\text {est }}\right)$, os resultados de abundância foram tabelados na forma de matriz de presença (1) e ausência (0). Em seguida, utilizouse o estimador Chao 2. Os dados foram calculados com o auxílio do programa EstimateS versão 7.5.2 (COLWELL, 2009), com 100 aleatorizações. Adotou-se o fator tempo (datas de coletas), para atender ao critério de independência entre as amostras.

A eficiência amostral verificada para a área de cada tratamento resultou da porcentagem (\%) da riqueza observada $\left(S_{\text {obs }}\right)$ em relação à riqueza estimada $\left(S_{\text {est }}\right)$.

Para avaliar a diversidade e a similaridade de espécies de formigas encontradas nas áreas dos diferentes tipos de controle de plantas infestantes, utilizaram-se, respectivamente, segundo Magurran (2011), uma medida não paramétrica de diversidade $\alpha$, calculada a partir do índice de diversidade de Shannon $\left(H^{\prime}\right)$, obtido através do programa DivEs versão 2.0 (RODRIGUES, 2005); e uma medida quantitativa de diversidade $\beta$, através do cálculo do índice de similaridade de Morisita-Horn (Imh), assim como uma análise de agrupamentos com a média não ponderada dos grupos de pares (Unweighted ParGroup Mean Average - UPGMA), como distância de amalgamação, através do programa estatístico Past (HAMMER; HARPER; RYAN, 2001).

\section{Identificação e quantificação de plantas infestantes}

Os levantamentos das espécies de plantas infestantes foram realizados antes do plantio (março de 2011) e seis meses após o plantio das mudas de Eucalyptus grandis (fevereiro de 2012). Para tanto, foram retiradas amostras da vegetação de cinco áreas com um metro quadrado $\left(\mathrm{m}^{2}\right)$ cada, por tratamento, selecionadas aleatoriamente. Os exemplares coletados foram encaminhados para identificação.

\section{Análise estatística}

Para a comparação da abundância e diversidade de espécies de formigas, realizouse análise de variância (ANOVA). Tendo como variáveis dependentes, para a abundância, os valores totais de formigas, e para a diversidade de espécies, os índices de diversidade de Shannon $\left(H^{\prime}\right)$ encontrados nos três métodos de coleta, para os períodos: antes da aplicação (março de 2011), após a primeira aplicação (de 01/04 até 29/06/2011), após a segunda aplicação (de 30/06 até 20/10/2011) e após a terceira aplicação dos herbicidas (21/10/2011 até 16/02/2012). E, como variáveis independentes, os tratamentos constituídos pelos diferentes tipos de controle de plantas infestantes.

A partir dos resultados da ANOVA, realizou-se o teste de médias através do Teste $t$, com $5 \%$ de probabilidade de erro. Sendo que os valores de abundância foram transformados, para atender aos critérios da estatística descritiva.

Para análise das relações entre a riqueza de espécies de plantas infestantes com a riqueza observada de espécies de formigas, utilizou-se correlação linear de Pearson.

A análise da variância, o teste de médias e a correlação de Pearson foram calculados utilizandose o programa estatístico Assistat 7.6 beta, desenvolvido por Silva e Azevedo (2009).

\section{RESULTADOS E DISCUSSÃO}

No período de março de 2011 a fevereiro de 2012, coletaram-se, em função dos três métodos de amostragem, um total de 46.675 espécimens de formigas, em um plantio inicial de Eucalyptus grandis Hill ex Maiden, submetido a diferentes sistemas de controle de plantas infestantes. As formigas foram classificadas em sete subfamílias, 14 tribos, 18 gêneros e 37 espécies, conforme pode ser observado na Tabela 1.

$\mathrm{O}$ número de formigas capturado $\mathrm{e}$ apresentado na Tabela 1 foi superior ao registrado por Fonseca e Diehl (2004), que foi de 21.033, em plantios de eucalipto, com diferentes idades, no Rio Grande do Sul. Possivelmente, a disparidade do número de indivíduos em comparação ao presente estudo, pode ter ocorrido devido aos períodos distintos de coleta, a frequência de amostragem ou aos diferentes métodos utilizados pelos autores, bem como a própria composição local das comunidades de formigas.

Não se verificou diferença significativa (ANOVA, $\mathrm{p}>=0,05$ ) entre os valores médios gerais do número de espécimens de formigas capturados nas áreas com aplicação dos diferentes tratamentos. No entanto, na área onde foi realizada a aplicação de glifosato em faixas, com o controle químico total de plantas infestantes em faixa de um metro paralela 
à linha de plantio e de um metro na parte central da entrelinha, sem controle, foram coletadas 12.842 formigas, correspondendo a $27,5 \%$ do total. Seguida das áreas: com controle de monocotiledôneas na linha e na entrelinha do plantio, com 9.243 (19,8\%); com controle químico total, na linha e entrelinha do plantio, com $8.472(18,1 \%)$; nas quais não houve nenhuma intervenção, com 5.736 (12,3\%); com controle químico total somente na linha de plantio, com 5.544 (11,9\%); e, por fim, pela área em que foi realizado o controle de dicotiledôneas na linha e na entrelinha do plantio, que apresentou a menor abundância, com 4.838 formigas $(10,4 \%)$ (Tabela 1).

Observou-se que as áreas com menor interferência da ação dos herbicidas sobre a composição florística original, nas quais foram instalados os tratamentos T6, T2 e T4, em termos de abundância de espécimens de formigas, demonstraram valores inferiores a menos da metade do número de formigas capturadas na área onde foi aplicado o tratamento $\mathrm{T} 5$ e distantes dos valores observados para as áreas dos tratamentos $\mathrm{T} 1 \mathrm{e} \mathrm{T} 3$ (Tabela 1).

Segundo Vasconcelos (1998), níveis mais elevados de perturbação ambiental resultam em um aumento da abundância de formigas, com predomínio de algumas espécies. Sendo assim, os resultados observados nas áreas correspondentes à instalação dos tratamentos $\mathrm{T} 5, \mathrm{~T} 1 \mathrm{e} \mathrm{T}$, quanto ao número de formicídeos coletados, podem estar relacionados com as alterações causadas na estrutura da vegetação local, pelos diferentes sistemas de controle de plantas infestantes através do uso de herbicidas.

Quanto à riqueza de espécies, a subfamília Myrmicinae foi encontrada em todas as áreas e, apresentou a maior riqueza de espécies $\left(S_{\mathrm{obs}}=21\right)$ (Tabela 1). Essa predominância pode ser explicada pelo fato de esta subfamília ser a mais abundante e apresentar a maior diversidade de hábitos na região Neotropical e no mundo (FOWLER et al., 1991).

Em meio aos gêneros identificados para Myrmicinae, Pheidole apresentou a maior riqueza de espécies observadas (Sobs $=6$ ). Cabe ressaltar que o gênero Pheidole é o mais abundante entre as espécies de formigas, tanto em número de colônias, quanto em número de indivíduos (WILSON, 1986). Trabalhos realizados em eucaliptais no Brasil, indicaram o gênero como tendo o maior número de espécies de formigas observadas (MARINHO et al., 2002; FONSECA e DIEHL, 2004; RAMOS-
LACAU et al., 2008; BOSCARDIN et al., 2011).

Dentre as espécies identificadas para Pheidole, destaca-se Pheidole pullula (Santschi, 1911), que ocorreu em todas as áreas avaliadas (Tabela 1). Pouco se sabe a respeito da ecologia desta espécie, sendo conhecida nas regiões de Córdoba e Buenos Aires, Argentina (DELABIE, 2012).

Pertencentes à subfamília Myrmicinae, tribo Attini, as formigas cortadeiras, representadas pelos gêneros Atta e Acromyrmex, apresentaram, respectivamente, $S_{\mathrm{obs}}=1$ e $S_{\mathrm{obs}}=4$. Não houve registros de Acromyrmex ambiguus (Emery, 1888) na área correspondente ao tratamento T6, nem de Acromyrmex striatus (Roger, 1863) nas áreas dos tratamentos T4 e T5 (Tabela 1).

Mycocepurus goeldii (Forel, 1893), também pertencente à tribo Attini, foi encontrada em todas as áreas avaliadas (Tabela 1). Em estudo realizado por Ramos et al. (2004), Mycocepurus goeldii foi sensível apenas à capina mecânica, que, provavelmente, segundo os autores, alterou seu nicho ecológico, não sendo suscetível à aplicação do herbicida glifosato.

A espécie Crematogaster victima Fr. (Smith, 1858) e Pogonomyrmex naegelii (Emery, 1878), também pertencentes à Myrmicinae, estiveram presentes em todas as áreas, enquanto a Crematogaster sp.1 não foi encontrada, apenas, na área onde se aplicou glifosato na linha e na entrelinha do plantio (Tabela 1). Segundo Silvestre e Silva (2001), espécies de Crematogaster caracterizam-se por nidificarem, principalmente, sobre a vegetação e apresentam hábito alimentar onívoro.

Strumigenys schulzi (Emery, 1894), ocorreu somente nas áreas dos tratamentos T2 e T4 (Tabela 1). De acordo com Fowler et al. (1991), espécies pertencentes à tribo Dacetini habitam o húmus intersticial e se alimentam, principalmente, de espécies pertencentes à ordem Collembola.

A segunda maior riqueza de espécies foi encontrada para a subfamília Formicinae $\left(S_{\mathrm{obs}}=7\right)$. As espécies desta subfamília se caracterizam por secretar ácido fórmico para sua defesa (JAFFÉ, 1993), compreendendo 49 gêneros viventes no mundo (FERNÁNDEZ, 2003). Dentro desta subfamília, prevaleceu o gênero Camponotus, com seis espécies. Dentre estas, destaca-se a espécie Camponotus punctulatus (Mayr, 1868), devido aos altos valores de abundância verificados nas áreas dos tratamentos T1, T3 e T5 (Tabela 1). Esta espécie se estabelece, especialmente, em áreas de pousio, após o cultivo de arroz irrigado (SIMAS; COSTA; 
TABELA 1: Abundância e riqueza de espécies de formigas coletadas com isca atrativa, armadilha de solo e funil de Berlese, em uma área com plantio inicial de Eucalyptus grandis, submetido a diferentes tipos de controle de plantas infestantes, em Santa Maria - RS. Março de 2011 a fevereiro de 2012.

TABLE 1: Abundance and species richness of ants collected with attractive bait, trap soil and Berlese funnel, in an area with initial planting of Eucalyptus grandis, subjected to different types of weed control in Santa Maria - RS. March 2011 to february 2012

\begin{tabular}{|c|c|c|c|c|c|c|}
\hline \multirow{2}{*}{ Subfamília/Tribo/Espécie } & \multicolumn{6}{|c|}{ Tratamentos $^{1}$} \\
\hline & T1 & T2 & T3 & $\mathrm{T} 4$ & T5 & T6 \\
\hline \multicolumn{7}{|l|}{ DOLICHODERINAE $\left(S_{\mathrm{obs}}^{2}=1\right)$} \\
\hline \multicolumn{7}{|l|}{ Dolichoderini } \\
\hline Dorymyrmex sp.1 & 1 & 2 & 11 & 4 & 460 & 12 \\
\hline \multicolumn{7}{|l|}{ ECITONINAE $\left(S_{\mathrm{obs}}=1\right)$} \\
\hline $\begin{array}{l}\text { Ecitonini } \\
\text { Labidus praedator (Fr. Smith, 1858) }\end{array}$ & 7 & 89 & 214 & 85 & 131 & 12 \\
\hline \multicolumn{7}{|l|}{ ECTATOMMINAE $\left(S_{\mathrm{obs}}=3\right)$} \\
\hline \multicolumn{7}{|l|}{ Ectatommini } \\
\hline Ectatomma edentatum Roger, 1863 & 1 & 5 & 1 & 8 & 1 & 1 \\
\hline Ectatomma permagnum Forel, 1908 & 19 & 51 & 3 & 3 & 3 & 163 \\
\hline Gnamptogenys sulcata (Fr. Smith, 1858) & 2 & 10 & 2 & 1 & - & 10 \\
\hline \multicolumn{7}{|l|}{ FORMICINAE $\left(S_{\mathrm{obs}}=7\right)$} \\
\hline \multicolumn{7}{|l|}{ Brachymyrmecini } \\
\hline $\begin{array}{l}\text { Brachymyrmex degener Emery, } 1906 \\
\text { Camponotini }\end{array}$ & 11 & 118 & 47 & 71 & 175 & 140 \\
\hline Camponotus blandus (Fr. Smith, 1858) & - & 1 & - & - & - & - \\
\hline Camponotus melanoticus Emery, 1894 & 1 & 2 & 2 & 8 & 5 & 1 \\
\hline Camponotus novogranadensis Mayr, 1870 & 74 & 2371 & 138 & 558 & 148 & 339 \\
\hline Camponotus punctulatus Mayr, 1868 & 3480 & 294 & 1495 & 26 & 2200 & 72 \\
\hline Camponotus rufipes Fabricius, 1775 & 54 & 160 & 3 & 216 & 16 & 1073 \\
\hline Camponotus senex (Smith, 1858) & - & 3 & - & 29 & - & - \\
\hline \multicolumn{7}{|l|}{ MYRMICINAE $\left(S_{\mathrm{obs}}=21\right)$} \\
\hline \multicolumn{7}{|l|}{ Attini } \\
\hline Acromyrmex ambiguus (Emery, 1888) & 62 & 32 & 5 & 1 & 5 & - \\
\hline Acromyrmex balzani (Emery, 1890) & 96 & 27 & 40 & 8 & 15 & 29 \\
\hline Acromyrmex heyeri Forel, 1899 & 37 & 11 & 15 & 8 & 2 & 5 \\
\hline Acromyrmex striatus (Roger, 1863) & 4 & 9 & 1 & - & - & 3 \\
\hline Atta sexdens piriventris Santschi, 1919 & 59 & 43 & 93 & 12 & 56 & 76 \\
\hline $\begin{array}{l}\text { Mycocepurus goeldii Forel, } 1893 \\
\text { Blepharidattini }\end{array}$ & 28 & 39 & 22 & 4 & 22 & 17 \\
\hline \multicolumn{7}{|l|}{ Crematogastrini } \\
\hline Crematogaster sp.1 & - & 20 & 10 & 3 & 16 & 23 \\
\hline Crematogaster victima Fr. Smith, 1858 & 5 & 2 & 2 & 1 & 178 & 176 \\
\hline \multicolumn{7}{|l|}{ Dacetini } \\
\hline $\begin{array}{l}\text { Strumigenys schulzi Emery, } 1894 \\
\text { Myrmicini }\end{array}$ & - & 2 & - & - & 1 & - \\
\hline $\begin{array}{l}\text { Pogonomyrmex naegelii Emery, } 1878 \\
\text { Pheidolini }\end{array}$ & 2 & 18 & 8 & 2 & 6 & 35 \\
\hline Pheidole complexo flavens sp.2 & 21 & 200 & 36 & 27 & 50 & 83 \\
\hline Pheidole grupo Tristis sp.1 & - & - & 1 & 27 & 1 & - \\
\hline Pheidole nesiota Wilson, 2003 & 22 & 57 & 43 & 136 & 22 & 43 \\
\hline Pheidole obscurithorax Naves, 1985 & 2201 & 796 & 3702 & 89 & 4487 & 1454 \\
\hline
\end{tabular}

Continua... 
TABELA 1: Continuação...

TABLE 1: Continued...

\begin{tabular}{|c|c|c|c|c|c|c|}
\hline \multirow{2}{*}{ Subfamília/Tribo/Espécie } & \multicolumn{6}{|c|}{ Tratamentos $^{1}$} \\
\hline & T1 & $\mathrm{T} 2$ & T3 & T4 & T5 & T6 \\
\hline Pheidole pullula Santschi, 1911 & 86 & 67 & 46 & 117 & 46 & 103 \\
\hline Pheidole radoszkowskii Mayr, 1884 & 80 & 523 & 227 & 766 & 341 & 597 \\
\hline \multicolumn{7}{|l|}{ Solenopsidini } \\
\hline Solenopsis invicta Buren, 1972 & 1848 & 235 & 2989 & 2540 & 4308 & 1074 \\
\hline Solenopsis sp.1 & 15 & 110 & 25 & 12 & 3 & 104 \\
\hline Solenopsis sp.2 & 4 & 23 & 2 & 3 & 4 & 1 \\
\hline Solenopsis sp.3 & 119 & 133 & 11 & 65 & 63 & 38 \\
\hline \multicolumn{7}{|l|}{ PONERINAE $\left(S_{\mathrm{obs}}=2\right)$} \\
\hline \multicolumn{7}{|l|}{ Ponerini } \\
\hline Hypoponera sp.1 & 2 & 1 & - & 2 & - & 9 \\
\hline Odontomachus chelifer (Latreille, 1802) & - & - & 1 & - & - & - \\
\hline \multicolumn{7}{|l|}{ PSEUDOMYRMECINAE $\left(S_{\mathrm{obs}}=2\right)$} \\
\hline \multicolumn{7}{|l|}{ Pseudomyrmecini } \\
\hline Pseudomyrmex grupo Pallidus sp.1 & 2 & 4 & - & 2 & - & - \\
\hline Pseudomyrmex grupo Pallidus sp. 2 & - & 2 & - & 1 & - & - \\
\hline Número de espécimens de formigas & 8472 & 5544 & 9243 & 4838 & 12842 & 5736 \\
\hline$S_{\text {obs }}$ & 30 & 35 & 31 & 33 & 29 & 29 \\
\hline
\end{tabular}

Em que: ${ }^{1}$ T1: controle químico total de plantas infestantes, na linha e entrelinha de plantio, com glifosato; T2: controle químico total de plantas infestantes na linha de plantio, com glifosato; T3: controle químico de monocotiledôneas na linha e entrelinha de plantio, com setoxidim; T4: controle químico de dicotiledôneas na linha e entrelinha de plantio, com bentazona; T5: controle químico total de plantas infestantes em faixa de um metro paralela à linha de plantio, com glifosato, e de um metro na parte central da entrelinha, sem controle; T6: testemunha, sem controle de plantas infestantes e nenhuma forma de limpeza da área. ${ }^{2} S_{\mathrm{obs}}$ : Riqueza observada de espécies de formigas.

SIMAS, 2000/2001), o que indica sua preferência por ambientes mais antropizados.

A partir desses resultados, pode-se inferir que exista uma relação entre a presença da espécie Camponotus punctulatus e o meio ambiente, pois a abundância mais elevada foi observada para a espécie na área em que foi instalado o tratamento T1, onde houve a simplificação da vegetação original, indicando assim, uma maior antropização. Por outro lado, no ambiente estruturalmente mais conservado, observado na área sem controle de matocompetição, Camponotus punctulatus apresentou apenas 72 indivíduos, seguido da área com controle de dicotiledôneas, com 26 espécimens coletados (Tabela 1).

O gênero Brachymyrmex, pertencente à Formicinae, está representado somente pela espécie Brachymyrmex degener (Emery, 1906), tendo sido capturada em todas as áreas dos tratamentos (Tabela 1), porém, capturada somente através do método de extração com funil de Berlese. Ramos et al. (2004), verificaram um efeito negativo para a espécie Brachymyrmex sp.1, juntamente com Solenopsis sp.1, Wasmannia auropunctata (Roger,
1863), após a aplicação de herbicida glifosato em área com eucalipto, o que não ocorreu no presente estudo. Porém, ressalta-se que a espécie de Brachymyrmex, encontrada pelos autores difere da observada no presente estudo, o que pode explicar a diferença na resposta comportamental das espécies.

A subfamília Ectatomminae, apresentou a terceira maior riqueza de espécies, com Ectatomma edentatum (Roger, 1863), Ectatomma permagnum apresentou 163 espécimens na área 1858) (Tabela 1). Sendo as duas primeiras tidas como espécies de formigas predadoras epígeas de grande tamanho por Silvestre, Brandão e Silva (2003), e a terceira como predadora generalista (DELABIE; AGOSTI; NASCIMENTO, 2000). Cabe ressaltar que Ectatomma permagnum (Forel, 1908) apresentou 163 espécimens na área sem controle de plantas infestantes (Tabela 1). A subfamília Ponerinae, apresentou duas espécies, sendo elas, Odontomachus chelifer (Latreille, 1802) e Hypoponera sp.1 (Tabela 1). Estas espécies estão também classificadas como predadora epígea de grande tamanho e generalista, respectivamente.

A subfamília Pseudomyrmicinae, assim 
como Ponerinae, apresentou somente duas espécies. Pseudomyrmex grupo Pallidus sp.1 capturada nas áreas em que foram instalados os tratamentos T1, T2 e T4, enquanto que Pseudomyrmex grupo Pallidus sp. 2 ocorreu somente nas áreas correspondentes aos tratamentos T2 e T4 (Tabela 1). Segundo Fowler et al. (1991), espécies pertencentes à Pseudomyrmicinae são arbóreas e territorialistas, possuem visão altamente discriminativa e rápida e são predadoras generalistas. Várias espécies mantêm associações mutualísticas obrigatórias com determinadas plantas, as quais dependem de fitófagos e das quais se obtêm alimento para complementar sua dieta. Pseudomyrmex termitarius é a única espécie comprovadamente terrícola e se alimenta de cupins.

Dorymyrmex sp.1, pertencente à subfamília Dolichoderinae, esteve presente em todas as áreas avaliadas (Tabela 1). Espécies de Dorymyrmex nidificam em áreas abertas com grama e possuem dieta à base de líquidos provenientes de plantas vivas, sendo que as operárias forrageiam sobre o solo (LONGINO, 2007).

Labidus praedator (Fr. Smith, 1858), pertencente à subfamília Ecitoninae, constituindose na única espécie representante desta subfamília, tendo sido registrada em todas as áreas avaliadas (Tabela 1). Segundo Brandão, Silva e Delabie (2009), trata-se de uma espécie epígea de hábito nômade, por isso chamada de formiga nômade, ou formiga de correição.

Quanto ao número de espécies capturadas na área de cada tratamento ao final das avaliações, conforme demonstra a Tabela 1, a área com controle químico realizado somente na linha de plantio (T2) apresentou, ao final do período de coleta, a maior riqueza de espécies observadas, com $S_{\text {obs }}=35$, em detrimento das demais; seguida pela área com controle químico de dicotiledôneas (T4), com Sobs $=33$ e da área com controle químico de monocotiledôneas (T3), com Sobs $=31$, ambos, T4 e T3, com controle na linha e entrelinha do plantio; e da área com controle químico total de plantas infestantes, tanto na linha quanto na entrelinha de plantio (T1), com $S_{\mathrm{obs}}=30$. Já as áreas em que foram aplicados os tratamentos com controle químico total de plantas infestantes em faixa de um metro paralela à linha de plantio e de um metro na parte central da entrelinha, sem controle (T5), e o testemunha, sem nenhuma intervenção (T6), apresentaram, ambas, a menor riqueza observada, com $\mathrm{S}_{\mathrm{obs}}=29$. Fonseca e Diehl (2004) encontraram valores semelhantes
$\left(\mathrm{S}_{\text {obs }}=35\right.$ e 37), em eucaliptais, com cinco anos de idade, no Rio Grande do Sul.

A partir da diferença entre os valores de riqueza observada e estimada, verificou-se que a eficiência amostral da área com controle químico total de plantas infestantes, correspondente ao tratamento $\mathrm{T} 1$, foi menor quando comparada às demais áreas avaliadas, com 93,5\% das espécies amostradas. Em contrapartida, para a área com controle químico total de plantas infestantes somente na linha de plantio (T2), verificou-se que o número de espécies amostrado foi mais próximo ao número estimado, com 99,0\%.

A área com controle químico de plantas infestantes em faixa de um metro paralela à linha de plantio e de um metro na parte central da entrelinha, sem controle, apresentou 98,7\% das espécies de formigas amostradas, seguida da área em que foi instalado o tratamento T3, com controle químico de monocotiledôneas na linha e entrelinha de plantio, com $98,1 \%$. Já a área sem intervenção apresentouse como a quarta mais eficiente, com $96,7 \%$, e a área correspondente ao tratamento $\mathrm{T} 4$, com controle químico de dicotiledôneas na linha e entrelinha do plantio, apresentou $95,9 \%$ das espécies de formigas amostradas.

Levando-se em consideração o número total de espécies de formigas observado e estimado, verifica-se uma pequena variação entre a eficiência amostral entre a área em que foi aplicado glifosato na linha e na entrelinha de plantio, em comparação à área em que não foi realizada nenhuma intervenção, sendo que, nessa última, foi observada a menor riqueza de espécies de formigas. Este fato sugere que a composição de espécies de formiga pode responder melhor às alterações entre os diferentes sistemas de controle de plantas infestantes do que a riqueza de espécies. Corroborando assim Schmidt e Diehl (2008), quando afirmam que o uso do solo afeta mais a composição de espécies do que a riqueza de espécies de formigas.

No entendimento de Bestelmeyer et al. (2000), a eficiência de amostragem pode ser influenciada por vários fatores, dentre os quais se destacam o método de amostragem empregado, composição da comunidade de formigas, o relevo e a heterogeneidade espacial. Nesse sentido, Ramos et al. (2004), ao avaliarem a diversidade de Shannon para a comunidade de formigas em Eucalyptus grandis, submetida à capina química com utilização de glifosato, verificaram uma diminuição nos valores do índice de Shannon oito dias depois da aplicação 
do herbicida, bem como um retorno aos valores similares iniciais encontrados antes das aplicações. Isso indica, segundo os autores, não ter havido um grande distúrbio por parte dos tratamentos sobre a diversidade de formigas.

No presente estudo, não houve diferença significativa (ANOVA, $\mathrm{p}>=0,05$ ) entre os valores médios dos índices de diversidade de Shannon $\left(H^{\prime}\right)$ observados nos diferentes sistemas de controle de plantas infestantes. Os índices de diversidade de Shannon foram, em ordem decrescente, para as áreas dos tratamentos: T2 $\left(H^{\prime}=1,34\right), \mathrm{T} 6\left(H^{\prime}=\right.$ $1,33)$, T4 $\left(H^{\prime}=1,30\right), \mathrm{T} 3\left(H^{\prime}=1,28\right), \mathrm{T} 1\left(H^{\prime}=1,25\right)$, e por fim $\mathrm{T} 5\left(H^{\prime}=1,23\right)$.

Os valores resultantes da eficiência amostral e índice de diversidade de Shannon observados para a área onde foi instalado o tratamento $\mathrm{T} 1$, em detrimento das demais áreas indicam uma possível simplificação do meio ambiente, através da modificação da fauna de formigas. Os dados apresentados na Tabela 2 confirmam tal simplificação em termos florísticos, devido à ação do herbicida pós-emergente glifosato, aplicado na linha e na entrelinha do plantio inicial de Eucalyptus grandis.

Nesta área, somente duas espécies de plantas infestantes, ou seja, $10,0 \%$ do total foram observadas na área ao final do período do experimento (fevereiro de 2012). As espécies foram identificadas como Solanum sisymbriifolium Lam

TABELA 2: Plantas infestantes presentes no final do período de avaliação do experimento (fevereiro de 2012), em plantio inicial de Eucalyptus grandis, submetido a diferentes tipos de controle de plantas infestantes, em Santa Maria - RS. Março de 2011 a fevereiro de 2012.

TABLE 2: Weeds present at the end of the evaluation period of the experiment (February 2012), in initial planting of Eucalyptus grandis, subjected to different types of weed control in Santa Maria RS. March 2011 to february 2012.

\begin{tabular}{|c|c|c|c|c|c|c|c|}
\hline \multirow{2}{*}{ Família/Espécie } & \multirow{2}{*}{ Nome Popular } & \multicolumn{6}{|c|}{ Tratamentos $^{1}$} \\
\hline & & $\mathrm{T} 1$ & $\mathrm{~T} 2$ & $\mathrm{~T} 3$ & $\mathrm{~T} 4$ & T5 & T6 \\
\hline APIACEAE & & & & & & & \\
\hline Eryngium horridum Malme & Gravatá & - & $\mathrm{x}$ & - & - & $\mathrm{x}$ & $\mathrm{X}$ \\
\hline $\begin{array}{l}\text { Centella asiatica (L.) Urb. } \\
\text { ASTERACEAE }\end{array}$ & Dinheiro-em-penca & - & $\mathrm{x}$ & $\mathrm{x}$ & - & $\mathrm{x}$ & $\mathrm{X}$ \\
\hline Baccharis sp. & Carqueja & - & $\mathrm{x}$ & - & $\mathrm{x}$ & $\mathrm{x}$ & $\mathrm{x}$ \\
\hline Conyza sp. & Buva & $\mathrm{x}$ & $\mathrm{x}$ & $\mathrm{x}$ & - & $\mathrm{x}$ & $\mathrm{x}$ \\
\hline Elephantopus mollis Kunth. & Suçuaia & - & $\mathrm{x}$ & - & - & $\mathrm{x}$ & $\mathrm{x}$ \\
\hline Pterocaulon alopecuroides (Lam.) DC. & Calção-de-velha & - & $\mathrm{x}$ & - & - & $\mathrm{x}$ & $\mathrm{x}$ \\
\hline Vernonia nudiflora Less. & Alecrim-do-campo & - & $\mathrm{x}$ & $\mathrm{x}$ & - & $\mathrm{x}$ & $\mathrm{x}$ \\
\hline Vernonia sp. & Mata-campo & - & $\mathrm{x}$ & $\mathrm{x}$ & - & $\mathrm{x}$ & $\mathrm{X}$ \\
\hline sp. & - & - & $\mathrm{x}$ & - & - & $\mathrm{x}$ & $\mathrm{X}$ \\
\hline sp. & - & - & $\mathrm{x}$ & - & - & $\mathrm{x}$ & $\mathrm{X}$ \\
\hline $\begin{array}{l}\text { Hyptis sp. } \\
\text { MALVACEAE }\end{array}$ & Falsa hortelã & - & $\mathrm{x}$ & $\mathrm{x}$ & - & $\mathrm{x}$ & $\mathrm{X}$ \\
\hline $\begin{array}{l}\text { Sida } \text { sp. } \\
\text { POACEAE }\end{array}$ & Guanxuma & - & $\mathrm{x}$ & - & - & $\mathrm{x}$ & $\mathrm{x}$ \\
\hline Panicum sp. 1 & Gramínea & - & $\mathrm{x}$ & - & $\mathrm{x}$ & $\mathrm{x}$ & $\mathrm{x}$ \\
\hline Panicum sp. 2 & Gramínea & - & $\mathrm{x}$ & - & $\mathrm{x}$ & $\mathrm{x}$ & $\mathrm{x}$ \\
\hline Piptochaetium montevidense (Spreng.) & Cabelo-de-porco & - & $\mathrm{x}$ & - & $\mathrm{x}$ & - & $\mathrm{x}$ \\
\hline Setaria geniculata (Lam.) P. Beauv. & Capim-rabo-de-raposa & - & $\mathrm{x}$ & - & - & - & $\mathrm{x}$ \\
\hline Sorghastrum sp. & Sorgo-do-campo & - & $\mathrm{x}$ & - & $\mathrm{x}$ & - & $\mathrm{x}$ \\
\hline $\begin{array}{l}\text { sp. } \\
\text { SOLANACEAE }\end{array}$ & Gramínea & - & $\mathrm{x}$ & - & - & $\mathrm{x}$ & $\mathrm{x}$ \\
\hline Solanum sisymbriifolium Lam. & Juá & $\mathrm{x}$ & $\mathrm{x}$ & $\mathrm{x}$ & - & $\mathrm{x}$ & $\mathrm{x}$ \\
\hline Solanum sp. & Fumo-bravo & - & $\mathrm{x}$ & - & - & - & $\mathrm{X}$ \\
\hline Número de espécies infestantes & 20 & 2 & 20 & 6 & 5 & 16 & 20 \\
\hline
\end{tabular}

Em que: ${ }^{1}$ T1: controle químico total de plantas infestantes, na linha e na entrelinha de plantio, com glifosato; T2: controle químico total de plantas infestantes na linha de plantio, com glifosato; T3: controle químico de monocotiledôneas na linha e na entrelinha de plantio, com setoxidim; T4: controle químico de dicotiledôneas na linha e na entrelinha de plantio, com bentazona; T5: controle químico total de plantas infestantes em faixa de um metro paralela à linha de plantio, com glifosato, e de um metro na parte central da entrelinha, sem controle; T6: testemunha, sem controle de plantas infestantes e nenhuma forma de limpeza da área. 
(juá) e Conyza sp. (buva).

De acordo com o observado na Tabela 2, a área em que foi realizado o controle de dicotiledôneas na linha e na entrelinha de plantio, apresentou-se como o segundo ambiente mais alterado, com cinco espécies remanescentes $(25,0 \%)$, seguido da área na qual foi realizado o controle de monocotiledôneas na linha e na entrelinha do plantio, com seis espécies $(30,0 \%)$.

A área com aplicação de glifosato em faixas, com controle da faixa de um metro paralela à linha de plantio e de um metro na parte central da entrelinha, sem controle, apresentou 16 espécies de plantas infestantes $(80,0 \%)$. Já as áreas com aplicação de glifosato somente na linha de plantio, na área em que não houve nenhuma intervenção sobre as plantas infestantes, mantiveram as 20 espécies de plantas indesejáveis $(100,0 \%)$ identificadas no pré- plantio. Não houve correlação $(r)$ significativa entre a riqueza total observada de espécies de formigas encontradas nos diferentes tipos de controle de plantas infestantes e a riqueza de espécies de plantas infestantes $(r=0,0463)$.

A partir do índice de Morisita-Horn (Imh), constatou-se que houve maior similaridade, ou seja, uma coexistência de espécies de formigas de maior abundância, entre as áreas em que foram instalados os tratamentos T3, T5 e T1, bem como maior similaridade entre as áreas dos tratamentos $\mathrm{T} 2, \mathrm{~T} 4$ e T6, conforme se pode observar na Figura 1.

A maior similaridade entre as formações estruturais de vegetação menos alteradas apresentou-se entre as áreas dos tratamentos $\mathrm{T} 2$ versus $\mathrm{T} 4(\operatorname{Imh}=0,92)$, seguidas das áreas de T4 versus $\mathrm{T} 6(\operatorname{Imh}=0,74)$ e $\mathrm{T} 2$ versus $\mathrm{T} 6(\operatorname{Imh}=$ $0,59)$, em contrapartida, nas formações estruturais

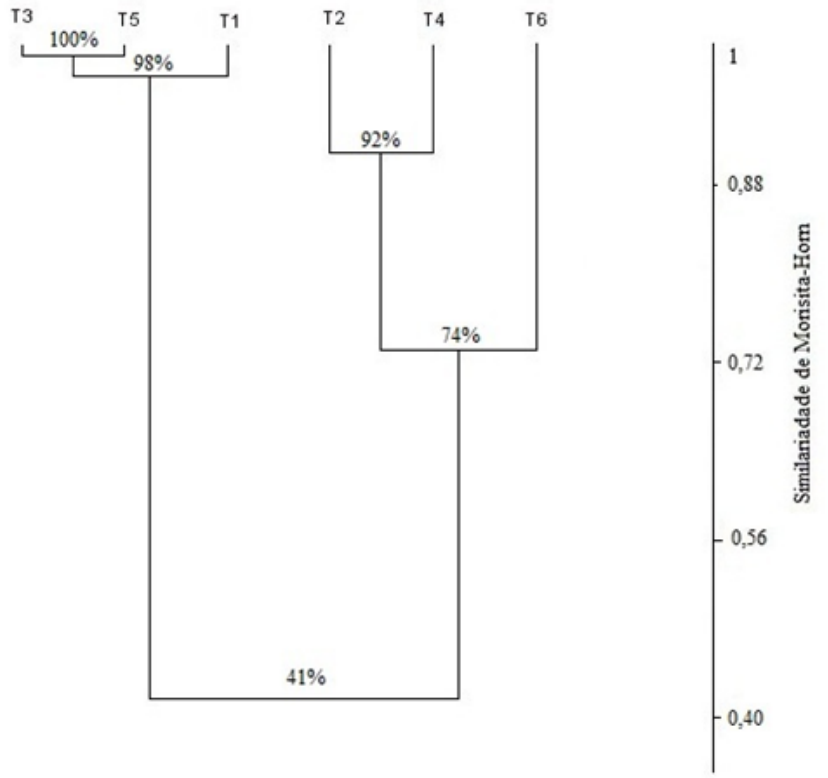

FIGURA 1: Dendrograma produzido a partir da análise de agrupamento utilizando a similaridade da composição específica de formigas obtida pelo índice de Similaridade de Morisita-Horn (Imh), entre as áreas com aplicação de glifosato, na linha e entrelinha do plantio (T1); aplicação de glifosato somente na linha de plantio (T2); aplicação de setoxidim na linha e entrelinha de plantio (T3); aplicação de bentazona na linha e entrelinha de plantio (T4); aplicação de glifosato em faixa de um metro paralelo à linha de plantio, e um metro na parte central da entrelinha, sem controle (T5); e testemunha, sem controle de plantas infestantes (T6), em um plantio inicial de Eucalyptus grandis, Santa Maria - RS. Março de 2011 a fevereiro de 2012.

FIGURE 1: Dendrogram produced from cluster analysis using a similarity in the species composition of ants obtained by similarity index of Morisita-Horn ( $\operatorname{Imh})$, among areas with glyphosate application, line spacing and planting (T1); glyphosate application only in the rows (T2); sethoxydim line spacing and planting (T3); application of bentazone in row spacing and planting (T4); glyphosate application in range of a meter parallel to the row, and one meter in the central part of the leading, without control (T5) and control without weed control (T6) in an initial planting of Eucalyptus grandis, Santa Maria - RS. March 2011 to february 2012. 
mais simplificadas, foi observada entre as áreas dos tratamentos T3 versus T5 $(\operatorname{Imh}=1,00)$, seguidas pelas áreas de $\mathrm{T} 1$ versus $\mathrm{T} 3 \mathrm{e} \mathrm{T} 1$ versus $\mathrm{T} 5$, ambas com valores iguais $(\operatorname{Imh}=0,98)$ (Figura 1$)$.

Cabe ressaltar que, apesar de a área em que foi instalado o tratamento T4 apresentar somente cinco espécies infestantes ao final do período de avaliação, esta se consistiu do controle somente de dicotiledôneas, com bentazona, mantendo, assim, o ambiente mais próximo ao natural (campo nativo), com a permanência das espécies de gramíneas (Tabela 2), fator que pode ter conservado a riqueza de espécies de formigas similar aos valores encontrados nos ambientes mais heterogêneos em termos de composição florística, constituídos pelas áreas dos tratamentos T2 e T6.

\section{CONCLUSÃO}

A composição de espécies de formigas apresenta sensibilidade às mudanças locais do ambiente, enquanto que a riqueza é pouco comprometida pelos efeitos indiretos dos diferentes tipos de controle de plantas infestantes, em um plantio inicial de Eucalyptus grandis.

\section{AGRADECIMENTOS}

Agradecemos ao Dr. Jacques Hubert Charles Delabie do Centro de Pesquisas do Cacau de Ilhéus na Bahia, pela identificação das espécies de formigas. Ao professor Dr. Sérgio Luiz de Oliveira Machado da Universidade Federal de Santa Maria, pela identificação das plantas infestantes. E à Coordenação de Aperfeiçoamento de Pessoal de Nível Superior (CAPES), pelo aporte financeiro através da concessão de bolsa de estudos ao primeiro autor.

À Fundação Estadual de Pesquisa Agropecuária (FEPAGRO), Unidade de Pesquisa Florestal de Santa Maria, RS, por disponibilizar a área de implantação do experimento e aos seus funcionários, pelo valioso auxílio.

\section{REFERÊNCIAS BIBLIOGRÁFICAS}

ABRAF. Associação Brasileira de Produtores de Florestas Plantadas. Anuário Estatístico da ABRAF 2012: ano base 2011. Disponível em: $<$ http://www.abraflor.org.br/estatisticas/ABRAF12/ ABRAF12-BR.pdf $>$. Acesso em: 05 set. 2012.

ALONSO, L. E. Ants as Indicators of Diversity. In:
AGOSTI, D. et al. (Ed.). Ants: standard methods for measuring and monitoring biodiversity. Washington and London: Smithsonian Institution Press, 2000, cap. 6, p. 80-88.

ALTIERI, M. A.; SILVA, E. do N.; NICHOLLS, C. I. O papel da biodiversidade no manejo de pragas. Ribeirão Preto: Holos, 2003. 226 p.

BESTELMEYER, B. T. et al. Fiel techiniques for the study of ground-dwelling ants: an overview, description, and evaluation. In: AGOSTI, D. et al. (Ed.). Ants: standard methods for measuring and monitoring biodiversity. Washington and London: Smithsonian Institution Press, 2000, cap. 9, p. 122-144.

BOLTON, B. Identification guide to the ant genera of the world. Cambridge: Harvard University Press, 1994. $222 \mathrm{p}$.

BOSCARDIN, J. et al. Avaliação comparativa de iscas atrativas a partir da riqueza de espécies de formigas (Hymenoptera: Formicidae) numa floresta de Eucalyptus grandis, em Santa Maria, Rio Grande do Sul, Brasil. AUGMDomus, Montevideo, v. 3, p.10-19, 2011.

BRANDÃO, C. R. F.; SILVA, R. R.; DELABIE, J. H. C. Formigas (Hymenoptera). In: PANIZZI, A. R.; PARRA, J. R. R. (Ed.). Bioecologia e nutrição de insetos: Base para o manejo integrado de pragas. Brasília: Embrapa Informação Tecnológica, 2009. cap. 9, p. 323-370.

COLWELL, R. K. EstimateS: statistical estimation of species richness and shared species from samples. Version 7.5.2. 2009. Disponível em: $<$ http://viceroy.eeb.uconn.edu/estimates $>$. Acesso em: 23 nov. 2011.

DELABIE, J. H. C. Pheidole pullula [mensagem pessoal]. Mensagem recebida por <boscardinj@ gmail.com> em 26 jun. 2012.

DELABIE, J. H. C.; AGOSTI, D.; NASCIMENTO, I. C. Litter ant communities of the Brazilian Atlantic rain forest region. In: AGOSTI, D. et al. (Ed.). Sampling ground-dwelling ants: case studies from the world's rain forests. Perth: Curtin University, 2000. cap. 1, p. 1-17 (Bulletin, 18).

FERNÁNDEZ, F. Subfamília Formicinae. In: FERNÁNDEZ, F. (Ed.). Introducción a las hormigas de la región Neotropical. Bogotá: Instituto de Investigación de Recursos Biológicos Alexander Von Humboldt, 2003. cap. 21, p. 299-306.

FERREIRA, L. R. et al. Manejo integrado de plantas daninhas na cultura do eucalipto. Viçosa: Editora da UFV, 2010. 140 p. 
FONSECA, R. C.; DIEHL, E. Riqueza de formigas (Hymenoptera, Formicidae) epigéicas em povoamentos de Eucalyptus spp. (Myrtaceae) de diferentes idades no Rio Grande do Sul, Brasil. Revista Brasileira de Entomologia, Curitiba, v. 48, n. 1, p. 95-100, mar. 2004.

FOWLER, H. G. et al. Ecologia nutricional de formigas. In: PANIZZI, A. R.; PARRA, J. R. R. (Ed.). Ecologia Nutricional de Insetos e Suas Implicações no Manejo Integrado de Pragas. São Paulo: Editora Manole LTDA, 1991, cap. 5, p. 131-223.

HAMMER, O.; HARPER, D.A.T.; RYAN, P. D. PAST: Paleontological Statistics Software Package for Education and Data Analysis. Palaeontologia Electronica, v. 4, n.1, p. 1-9, 2001.

HÖLLDOBLER, B.; WILSON, E. O. The ants. Cambridge: The Belknap Press of Harvard University Press, 1990. 732 p.

JAFFÉ, K. El mundo de las hormigas. Caracas: Equinoccio/ediciones de la Universidad Simón Bolívar, 1993. 190p.

LONGINO, J. T. Dorymyrmex. In: Ants of Costa Rica. Olympia: The Evergreen State College, 2007. Disponível em: <http://www.evergreen.edu/ants/ antsofcostarica.html >. Acesso em: 09 mar. 2012.

LOPES, C. T.; VASCONCELOS, H. L. Evaluation of three methods for sampling ground-dwelling ants in the brazilian cerrado. Neotropical Entomology, Londrina, v.37, n. 4, p.399-405, jul./aug. 2008.

MAGURRAN, A. E. Medindo a diversidade ecológica. Tradução Dana Moiana Vianna. Curitiba: Ed. da UFPR, 2011. 261 p.

MAJER, J. D. Ants: Bio-indicators of minesite rehabilitation, land-use, and land conservation. Environmental Management, New York, v. 7, n. 4, p. 375-383, 1983.

MARINHO, C. G. S. et al. Diversidade de Formigas (Hymenoptera: Formicidae) da Serapilheira em Eucaliptais (Myrtaceae) e Área de Cerrado de Minas Gerais. Neotropical Entomology, Londrina, v. 31, n. 2, p. 187-195, abr.jun. 2002.

MORENO, J. A. Clima do Rio Grande do Sul. Porto Alegre: Secretaria da Agricultura, 1961. 42 p. PERRANDO, E. R. Caracterização física e biológica do solo após aplicação de herbicidas em plantios de Acácia-Negra (Acacia mearnsii De Wild.) no Rio Grande Do Sul. 2008. 93 f. Tese (Doutorado em Engenharia Florestal) Universidade federal de Santa Maria, Santa Maria, 2008.

RAMOS, L. de S. et al. Impacto das capinas mecânica e química do sub-bosque de Eucalyptus grandis sobre a comunidade de formigas (Hymenoptera: Formicidae). Revista Árvore, Viçosa, v. 28, n.1, p. 139-146, 2004.

RAMOS-LACAU, L. de S. et al. Respostas das guildas de formigas (Hymenoptera: Formicidae) a práticas silviculturais em plantio de eucaliptos. Agrotrópica, Ilhéus, v. 20, p. 61-72, 2008.

RODRIGUES, W. C. DivEs - Diversidade de espécies. Versão 2.0. Software e Guia do Usuário, 2005. Disponível em: <http://www.ebras.bio.br>. Acesso em: 10 fev. 2010.

SARMIENTO-M, C. E. Metodologías de captura $\mathrm{y}$ estudio de las hormigas. In: FERNÁNDEZ, F. (Ed.). Introducción a las hormigas de la región Neotropical. Bogotá: Instituto de Investigación de Recursos Biológicos Alexander Von Humbolt, 2003. cap. 12, p. 201-210.

SCHMIDT, F. A.; DIEHL, E. What is the Effect of Soil Use on Ant Communities? Neotropical Entomology, Londrina, v. 37, n. 4, p. 381-388, jul./ago. 2008.

SILVA, F. de A. S.; AZEVEDO, C. A. V. de. Principal components analysis in the software Assistatstatistical attendance. In: WORLD CONGRESS ON COMPUTERS IN AGRICULTUMIDADE RELATIVAE, 7. Reno, USA: Anais... Reno, USA: American Society of Agriculal and Biological Engineers, 2009.

SILVESTRE, R.; BRANDÃO, C. R. F.; SILVA, R. R. Gremios funcionales de hormigas: El caso de lós gremios del Cerrado. In: FERNANDÉZ, F. (Ed.). Introducción a las hormigas de La región Neotropical. Bogotá: Fundación Humboldt, 2003. cap. 7, p. 113-148.

SILVESTRE, R.; SILVA, R. R. da. Guildas de formigas da Estação Ecológica Jataí, Luiz Antônio - SP. Sugestões para aplicação do modelo de guildas como bio-indicadores ambientais. Biotemas, Florianópolis, v.14, n. 1, p.37-69, 2001.

SIMAS, V. R.; COSTA, E. C.; SIMAS, C. A. Controle de Camponotus punctulatus Mayr, 1868 (HYMENOPTERA: FORMICIDAE). Revista de FZVA, Uruguaiana, v. $7 / 8$, n.1, p. 24-32, 2000/2001.

STRECK, E. V. et al. Solos do Rio Grande do Sul. 2. ed. Porto Alegre: Emater-RS/ASCAR, 2008. 222p.

TUFFI SANTOS, L. D. Efeitos diretos e indiretos do glyphosate em eucalipto. 2006. 80 f. Tese (Doutorado em Fitotecnia) - Universidade Federal de Viçosa, Viçosa, 2006. 
VASCONCELOS, H. L. Respostas das Biology \& Biochemistry, v. 33, n. 7, p.893-905, formigas a fragmentação florestal. Série 2001.

Técnica IPEF, Piracicaba, v.12, n. 32, p.95-98, WILSON, E. O. The defining traits of fire ants dez.1998.

WARDLE, D. A. et al. Impacts of ground vegetation and leaf-cutting ants. In: LOEFGREEN, C.S.; management strategies in a kiwifruit orchard on the VANDER MEER, R. K. (Ed.). Fire ants and leafcomposition and functioning of the soil biota. Soil cutting ants: biology and management. Boulder: Westview Press, 1986. cap.1. p.1-9.

Ci. Fl., v. 26, n. 1, jan.-mar., 2016 